\title{
Dimensiones estratégicas para el fortalecimiento de la responsabilidad social empresarial en Pymes del sector de la construcción en Colombia
}

\section{Strategic dimensions for strengthening corporate sociall responsibility in SMEs in the construction sector in Colombia}

\author{
Jose Morelos Gómez¹, Hernando José García Díaz², Mayra Prada Sánchez ${ }^{3}$
}

1 Director Maestría en Gestión de Organizaciones, docente titular de la Universidad de Cartagena, adscrito a la Facultad de Ciencias Económicas en el programa de Administración Industrial, Doctor en Ciencias Sociales - mención Gerencia Universidad del Zulia. Universidad de Cartagena, Piedra de Bolívar, Avenida del Consulado Calle 30 No. 48-152 Apartado Aéreo No. 1382, Cartagena, Colombia jmorelosg@unicartagena.edu.co

2 Ingeniero Civil, Universidad de Cartagena, Especialista en Gerencia de Proyectos de Construcción, Universidad de Cartagena, Cartagena, Colombia.

ing.garcia.diaz24@gmail.com

${ }^{3}$ Arquitecta, Universidad Jorge Tadeo Lozano, Especialista en Gerencia de Proyectos de Construcción, Universidad de Cartagena, Cartagena, Colombia.

mayra.prada.sanchez@gmail.com

Recibido: 15/feb/2020 Revisado: 30/abr/2020

Aceptado: 30/may/2020 Publicado: 30/jul/2020

Resumen Este artículo presenta una revisión de las dimensiones estratégicas para el fortalecimiento de políticas de responsabilidad social empresarial (RSE) en las Pymes del sector de la construcción en Colombia. La investigación fue de tipo descriptivo y explicativo, en la cual se aplicó instrumento estructurado in Situ a 45 Pymes seleccionadas del sector, para conocer el cumplimiento de lineamientos establecidos en la norma GTC-180, para los años 2017 y 2018. Como resultado, se encontró que aun cuando las empresas de estudio incluyen los conceptos de RSE en su organización, no califican y aplican todos los elementos valorados, respecto a los estándares establecidos por las instituciones que velan por el cumplimiento de los lineamientos de responsabilidad social establecidos.

Palabras claves responsabilidad social empresarial; pymes; sector construcción; buenas prácticas.

Abstract This article presents a review of the strategic dimensions for strengthening corporate social responsibility (CSR)
policies in SMEs in the construction sector in Colombia. The research was descriptive and explanatory, in which a
structured instrument was applied in situ to 45 selected SMEs in the sector, to find out the compliance with the guidelines
established in the GTC-180 standard, for the years 2017 and 2018 . As a result, found that even when the study
companies include the concepts of CSR in their organization, they do not qualify and apply all the elements valued, with
respect to the standards established by the institutions that ensure compliance with the established social responsibility
guidelines

Keywords corporate social responsibility; SMEs; construction sector; good practices.

Teknos Revista Científica. | Volumen 20 No.1 - Julio 2020 | ISSN 1900-7388 (papel) | ISSN 2539-2190 (digital)

DOI: https://doi.org/10.25044/issn.2539-2190 


\section{Introducción}

Desde la primera mitad del siglo $\mathrm{XX}$, distintas entidades a nivel nacional $e$ internacional, se han encargado de definir y establecer términos $y$ conceptos relacionados con la responsabilidad social empresarial RSE, puesto que para ese entonces, solo se planteaban soluciones a los problemas sociales que pudiesen presentarse en momentos específicos (Correa, 2007).

Es así como en la mitad siguiente del mismo siglo, la comunidad en general inicia un proceso de toma de conciencia, encabezados por el sector privado para influir y solucionar problemas sociales, lo cual generó que cada sistema estatal interviniera con un llamado al cumplimiento de normas que protegen los intereses públicos y los recursos naturales (Botero, 2009).

Lo anterior trajo consigo la necesidad en las organizaciones de contar con una política RSE, con el fin de avanzar en los propósitos de sostenibilidad, partiendo del hecho que exista una interacción de todos los ámbitos que pueden incurrir en la organización, desde el social y económico, hasta el ambiental. Además del individual, desde el punto de vista de cada trabajador. Según se ha citado en la Cumbre de Lisboa del 23 y 24 de marzo de 2000, la Unión Europea estableció para la siguiente década, el objetivo estratégico de convertirse en "la economía más competitiva y dinámica, basada en el conocimiento, capaz de un crecimiento económico sostenible con más y mejores empleos, y una mayor cohesión social", haciendo por primera vez un llamado expreso a las empresas en torno a su responsabilidad social. (Cumbre de Lisboa, 2000).

En este sentido, este artículo indaga la importancia de la RSE y su aplicación en las Pymes del sector de la construcción en Colombia, dado que los empresarios de este campo han esbozado mecanismos para comprender el concepto sobre responsabilidad social empresarial y han elaborado, del mismo modo, prácticas empresariales derivadas de dicho concepto, que moldean la condición laboral del trabajador en el país, el cual no debería estar ajeno a dicho debate, dado que es de vital importancia para el desarrollo económico y social de Colombia, por sus impactos en la generación de mano de obra y su efecto dinamizador en actividades conexas (Escuela de Negocios, 2005).

Para este estudio, se realizó una investigación de tipo descriptivo y explicativo, a partir de la identificación de las características de las 45 Pymes seleccionadas del sector construcción en Colombia, considerando la aplicación de los fundamentos de la RSE en el país. La estructura desarrollada para la presentación de este artículo, integra inicialmente los principales aportes del concepto de la RSE en el contexto internacional, seguidamente, se presentan los lineamientos definidos en la norma GTC-180, finalmente, se muestran los resultados comparativos de la aplicación y práctica que realizan las Pymes del sector de la construcción en Colombia en relación con los lineamientos de la RSE.

\section{Antecedentes}

\subsection{Acepción de la RSE}

La responsabilidad social empresarial RSE, es esencialmente un concepto con determinación, en la cual las empresas deciden voluntariamente contribuir al logro de una mejor sociedad, un medio ambiente limpio, e intenta determinar sus valores comunes tomando como base la Carta de los derechos fundamentales (Benítez, 2012). Al llegar a este punto, se puede decir que a la fecha, un número creciente de empresas reconoce cada vez más claramente su responsabilidad social y la considera parte de su identidad corporativa (CCRS, 2004). Esta responsabilidad se expresa frente a los trabajadores y en general, frente a todos los integrantes de la empresa, que pueden hacer parte de su cultura de éxito (Benbeniste, 2002, Morelos \& Fontalvo, 2014). En este sentido, las empresas deben asumir su responsabilidad social a nivel nacional e internacional 
(Fundación Entorno, 2009) a lo largo de toda su cadena de producción (Osorio, 2012).

En el campo ideológico, cuando se aborda el concepto de responsabilidad social, se pueden identificar dos tendencias, la primera considera la RSE como una actividad implícita de la empresa bajo la premisa que la organización puede obtener beneficios en tanto y en cuanto beneficie a la sociedad (Martí, Puerta \& Rojas, 2017), la segunda se basa en los postulados de los clásicos de la economía y en la idea de la mano invisible, la cual supone que por el solo hecho de haber empresas funcionando, la sociedad obtiene beneficios, lo que reduce el concepto de Responsabilidad Social Empresarial al comportamiento natural del mercado (Aguilera \& Puerto, 2012), es decir, una estrategia que se presenta no por iniciativa de la empresa si no por la misma naturaleza de la economía, sin embargo, este postulado riñe con algunos preceptos éticos y morales en el ámbito jurídico sobre todo porque las decisiones tomadas bajo este modelo, intentan cumplir con el interés último de la organización el cual es la obtención de beneficios, sin tratar de dañar los intereses de todos los demás actores implicados (trabajadores, gobierno, consumidores, etc.), y en el eventual caso de dañar los intereses de estos últimos, la empresa sería responsable, pero individualmente no habría responsabilidad, ya que los administradores están cumpliendo con la función de "desarrollar la sociedad"(Olano, 2014). En este sentido, Mababu (2010), afirma que RSE se refiere al acuerdo normativo empresarial, orientado al cumplimiento de los propósitos de la organización en materia social, económica y ambiental, de manera que el aporte de las partes, permita apalancar el crecimiento organizacional y la agregación de valor a todo su grupo de interés (CCE, 2001).

\subsection{Dimensiones de la RSE}

El concepto de Responsabilidad Social, abarca dimensiones o campos en los cuales las organizaciones ejercen alguna incidencia con sus actividades (Duque \& Martínez, 2012). La preocupación de los gerentes y directivos, está enfocada a la satisfacción de necesidades de cada uno de los implicados en las actividades de la organización, por lo tanto, un verdadero programa de RSE debe tener impactos sociales, medioambientales y económicos (Aparicio \& Valdés, 2009), como se describe a continuación:

- Dimensión económica: Se relaciona con las actividades orientadas a lograr una eficiente gestión económica, las buenas prácticas en los mercados financieros y de valores y las buenas relaciones con los grupos de interés. Asimismo, pretende consolidar una visión integral de RSE con las necesidades futuras de la sociedad y la forma cómo estas, podrían ser atendidas por las empresas (García, 2013).

- Dimensión socio-laboral: define el cumplimiento de las normas laborales internacionales y nacionales, a lo cual se le adicionan algunas actividades vinculadas con el rechazo a todo tipo de discriminación, a la vinculación laboral de grupos vulnerables de negociación colectiva y la consulta a los sindicatos y a la población en general (Camacho, 2015).

- Dimensión medioambiental: orientada al cumplimiento de la legislación medioambiental, manejo de residuos sólidos, reutilización de las materias primas, la integración social y cultural en temas medio ambientales (Grueso, 2008).

\subsection{Norma GTC - 180 en Colombia}

En Colombia se inició un proceso de implementación de normas que estipularan los puntos necesarios para la fundamentación de las bases de RSE en las empresas, el cual se ratifica con la publicación de las GTC - 180, de la Guía Técnica Colombiana (ICONTEC, 2008), la cual pretende direccionar las entidades hacia la mejora continua de las buenas prácticas con enfoques sociales, que involucren las partes interesadas. Esta debe ser aplicada por cualquier tipo de organización, sin tener en cuenta el tamaño, la razón social o el sector al que pertenezca. Su adopción es de carácter voluntario y 
no exime del cumplimiento de las disposiciones legales vigentes.

\section{Metodología}

El enfoque metodológico implementado en esta investigación, es de tipo descriptivo - explicativo, en la cual se identifican los elementos de la RSE a partir de un análisis racional y lógico, el cual permitió proponer nuevas acciones conducentes a la mejoraf de los procesos de los RSE en las Pymes del sector de la construcción. Asimismo, es explicativo dado que se busca exponer los resultados de la aplicación de la norma GTC- 180 a nivel nacional, en las 45 Pymes colombianas seleccionadas en el sector de la construcción, a fin de identificar las buenas prácticas de RSE implementadas en las pequeñas y medianas empresas de dicho sector en Colombia.

Para tal efecto, se emplearon los lineamientos establecidos en la norma GTC - 180 (ICONTEC, 2008) y en las metodologías de implementación de esta normativa y sus parámetros de aplicación en aquellas entidades que incorporan los criterios de sostenibilidad económica, social y ambiental en sus estrategias corporativas. Para tal efecto, se tuvo como base principal, el reconocimiento que lidera la Cámara Colombiana de la Construcción CAMACOL, "Premio a la Responsabilidad Social CAMACOL" (CAMACOL, 2019) que, bajo ciertos criterios de evaluación, exalta las diferentes actividades gestoras con alto impacto, empleadas por las empresas del sector. Finalmente, se proponen las estrategias para el mejoramiento de la aplicación de las RSE en la gestión organizacional de las Pymes del sector de la construcción en Colombia.

\section{Resultados}

De acuerdo al análisis de evaluación de la RSE utilizado, se obtuvo la estimación de 3 categorías principales, las cuales las Pymes del sector de la construcción, le apuntan a la implementación de la RSE, fortaleciendo las acciones conducentes al mejoramiento de las relaciones laborales, sociales y la responsabilidad ambiental, por lo cual CAMACOL, como entidad gestora de las iniciativas de la implementación de la normatividad, evalúa 3 diferentes programas descritos a continuación:

a) Gestión de la comunidad: Son todos los proyectos relacionados con las mejoras de las problemáticas sociales, fortalecimiento de las capacidades integrales, tales como ingresos, salud, educación, promoción de los derechos humanos, dinámicas para la economía local, etc.

b) Experiencias ambientales: Son aquellos programas de regulación del impacto ambiental, de acuerdo a la naturaleza de las actividades empresariales, como por ejemplo, los planes de mitigación, recuperación de zonas afectadas, o la promoción del desarrollo sostenible.

c) Prácticas de gestión socio-laboral: Son las actividades desarrolladas a nivel interior de las organizaciones, que tienen como objetivo mejorar la calidad de vida de las personas que la integran y/o sus familias, dignificando su nivel de vida.

A continuación, se muestra la evaluación al grupo de las 45 Pymes que se postularon en las distintas categorías, para competir por el premio a la Responsabilidad Social CAMACOL para los años 2017 y 2018, en el cual se evalúan las categorías que determinan las diferentes acciones a las que apuntan cada una las Pyme, con el propósito de cumplir con los lineamientos definidos en la norma GTC 180. Ver Tabla 1 y 2 , respectivamente.

Igualmente, se consideraron los siguientes criterios diferenciadores y de valor agregado, que impulsan el éxito de buenas prácticas de RSE en las Pymes, a saber: Impacto, participación de la comunidad o beneficiarios, sostenibilidad, replicabilidad de los proyectos, innovación, compromiso y core business, los cuales generan un incremento en la aplicación de las RSE.

Como resultados finales, a partir de los datos observados en la Tabla 1 y 2 , se tiene que para el 2017 , se evaluó un mayor número de Pymes constructoras con respecto al año siguiente, dónde se evidencia que, a pesar de la alta participación de las mismas, su porcentaje de aceptación fue inferior, lo cual determina que a pesar de que las empresas estén incluyendo los 
conceptos de RSE en su organización, no califican en los estándares establecidos por las instituciones que velan por su cumplimiento.

Tabla 1 Evaluación Pymes 2017

\begin{tabular}{lcc}
\hline \multicolumn{3}{c}{ Evaluación Pymes 2017 } \\
\hline \multicolumn{1}{c}{ Categoría } & $\begin{array}{c}\mathrm{N}^{\circ} \text { de } \\
\text { Pymes } \\
\text { que } \\
\text { emplean }\end{array}$ & $\begin{array}{c}\text { \% de } \\
\text { aceptación }\end{array}$ \\
\hline Gestión con la comunidad & 2 & $4 \%$ \\
\hline Experiencia ambiental & 2 & $4 \%$ \\
\hline $\begin{array}{l}\text { Gestión sociolaboral con las } \\
\text { personas que integran la } \\
\text { organización }\end{array}$ & 2 & $4 \%$ \\
\hline
\end{tabular}

Tabla 2 Evaluación Pymes 2018 Evaluación Pymes 2018

\begin{tabular}{|c|c|c|}
\hline \multicolumn{3}{|c|}{ Evaluación Pymes 2018} \\
\hline \multicolumn{3}{|c|}{ Total, Pymes evaluadas: 45} \\
\hline Categoría & $\begin{array}{l}\mathrm{N}^{\circ} \mathrm{de} \\
\text { pymes } \\
\text { que } \\
\text { emplean }\end{array}$ & $\begin{array}{c}\% \text { de } \\
\text { aceptación }\end{array}$ \\
\hline Gestión con la comunidad & 5 & $11,1 \%$ \\
\hline Experiencia ambiental & 4 & $8 \%$ \\
\hline $\begin{array}{l}\text { Gestión sociolaboral con las } \\
\text { personas que integran la } \\
\text { organización }\end{array}$ & 4 & $8 \%$ \\
\hline
\end{tabular}

Por otra parte, cabe anotar que el enfoque con mayor auge que se presenta en las prácticas de RSE en las Pymes constructoras colombianas, son los lineamientos de gestión con la comunidad, lo cual indica que el desarrollo social, es prioritario para el caso de las Pymes, dado que, es esta actividad donde se promueve la equidad, la inclusión social y acceso a oportunidades para el desarrollo de la población en general.

A continuación, se muestra la Figura 1, la cual define la interrelación entre los distintos enfoques de planteamiento de la estructuración de la metodología a implementar, de acuerdo a lo descrito anteriormente.

Por tanto, se propone a continuación la implementación de los componentes establecidos en las Tablas 3, 4 y 5, el cual plantea intervenir con el ciclo PHVA de la GTC-180 (Planear, Hacer, Verificar y Actuar), que recopile los criterios básicos mínimos, para el cumplimiento de los parámetros de RSE definidos por la norma, a través de los distintos componentes de gestión de la comunidad, ambiental y sociolaboral.

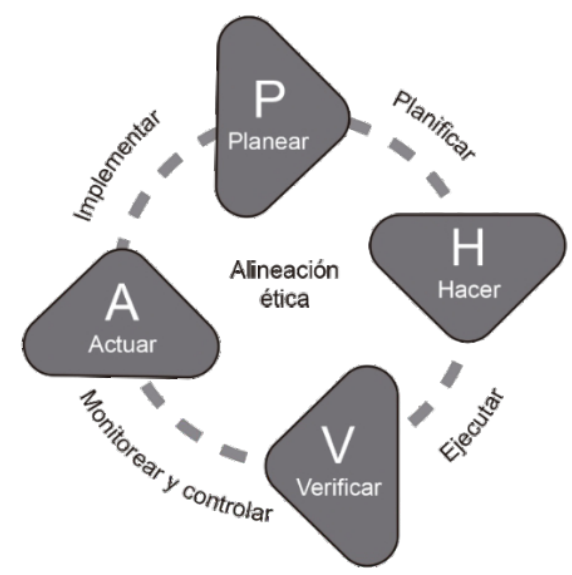

Figura 1. Ciclo de una gestión socialmente responsable

Tabla 3 Metodología para la gestión de la comunidad

\begin{tabular}{|c|c|c|c|}
\hline \multicolumn{4}{|c|}{ Componente: Gestión de la comunidad } \\
\hline Etapas & $\begin{array}{l}\text { Herramientas y } \\
\text { técnicas }\end{array}$ & Metodologías & $\begin{array}{l}\text { Resultados } \\
\text { esperados }\end{array}$ \\
\hline \multirow{4}{*}{$\begin{array}{l}\text { Planea- } \\
\text { ción }\end{array}$} & $\begin{array}{l}\text { Datos } \\
\text { poblacionales }\end{array}$ & $\begin{array}{l}\text { Muestreo } \\
\text { estadístico }\end{array}$ & $\begin{array}{l}\text { Definición de la } \\
\text { población } \\
\text { beneficiada con } \\
\text { el proyecto }\end{array}$ \\
\hline & $\begin{array}{l}\text { Necesidades a } \\
\text { satisfacer }\end{array}$ & $\begin{array}{l}\text { Estudio de las } \\
\text { problemáticas del } \\
\text { sitio }\end{array}$ & $\begin{array}{l}\text { Beneficios a } \\
\text { recibir }\end{array}$ \\
\hline & $\begin{array}{l}\text { Estrategias } \\
\text { para } \\
\text { mantenerlo }\end{array}$ & $\begin{array}{l}\text { Renovación de la } \\
\text { información } \\
\text { obtenida en los } \\
\text { análisis y } \\
\text { resultados }\end{array}$ & $\begin{array}{l}\text { Procesos para } \\
\text { renovar } \\
\text { periódicamente } \\
\text { el proyecto }\end{array}$ \\
\hline & $\begin{array}{l}\text { Definir los } \\
\text { lineamientos }\end{array}$ & $\begin{array}{l}\text { Establecer la } \\
\text { capacidad de } \\
\text { adaptabilidad del } \\
\text { proyecto }\end{array}$ & $\begin{array}{l}\text { Aplicable a } \\
\text { otras } \\
\text { poblaciones }\end{array}$ \\
\hline \multirow[t]{2}{*}{$\begin{array}{l}\text { Planea- } \\
\text { ción }\end{array}$} & Aportes & $\begin{array}{l}\text { Establecer } \\
\text { iniciativas propias } \\
\text { del lugar donde } \\
\text { se va a } \\
\text { implementar el } \\
\text { proyecto }\end{array}$ & $\begin{array}{l}\text { Necesidades } \\
\text { resueltas }\end{array}$ \\
\hline & $\begin{array}{l}\text { Fundamentació } \\
\text { n del proyecto }\end{array}$ & $\begin{array}{l}\text { Evaluaciones } \\
\text { continuas al } \\
\text { proyecto }\end{array}$ & $\begin{array}{l}\text { Renovación } \\
\text { constante de las } \\
\text { iniciativas } \\
\text { propuestas }\end{array}$ \\
\hline \multirow[b]{2}{*}{ Hacer } & $\begin{array}{l}\text { Inclusión de la } \\
\text { comunidad }\end{array}$ & $\begin{array}{l}\text { Vinculación al } \\
\text { proyecto }\end{array}$ & $\begin{array}{l}\text { Armonización } \\
\text { entre las partes } \\
\text { interesadas }\end{array}$ \\
\hline & $\begin{array}{l}\text { Objetivos del } \\
\text { proyecto }\end{array}$ & $\begin{array}{l}\text { Transferencia del } \\
\text { conocimiento, } \\
\text { generación de } \\
\text { capacidades }\end{array}$ & $\begin{array}{l}\text { Apropiación de } \\
\text { los resultados } \\
\text { por parte de los } \\
\text { beneficiarios }\end{array}$ \\
\hline
\end{tabular}


Morelos Gómez, J., García Díaz, H. J., \& Prada Sánchez, M. (2020). Dimensiones estratégicas para el fortalecimiento de la responsabilidad social empresarial en Pymes del sector de la construcción en Colombia. Teknos Revista

Científica, 20(1), 43-51.

\begin{tabular}{|c|c|c|c|}
\hline & $\begin{array}{l}\text { Definición del } \\
\text { alcance }\end{array}$ & $\begin{array}{l}\text { Ejecución de } \\
\text { todos los } \\
\text { entregables }\end{array}$ & $\begin{array}{l}\text { Cumplimiento del } \\
\text { alcance }\end{array}$ \\
\hline \multirow{3}{*}{ verificar } & $\begin{array}{l}\text { Monitoreo de la } \\
\text { participación de } \\
\text { la comunidad }\end{array}$ & $\begin{array}{l}\text { Realizar } \\
\text { revisiones } \\
\text { periódicas }\end{array}$ & $\begin{array}{l}\text { Registro de } \\
\text { verificación. } \\
\text { Datos de } \\
\text { participación }\end{array}$ \\
\hline & $\begin{array}{l}\text { Procesos de } \\
\text { sostenibilidad }\end{array}$ & $\begin{array}{l}\text { Evaluar los } \\
\text { procesos de } \\
\text { aplicación de } \\
\text { sostenibilidad }\end{array}$ & $\begin{array}{l}\text { Reporte del } \\
\text { cumplimiento de } \\
\text { la sostenibilidad } \\
\text { del proyecto }\end{array}$ \\
\hline & $\begin{array}{l}\text { Seguimiento } \\
\text { del } \\
\text { cumplimiento } \\
\text { de las } \\
\text { obligaciones }\end{array}$ & $\begin{array}{l}\text { Chequeos y } \\
\text { monitoreos } \\
\text { periódicos }\end{array}$ & $\begin{array}{l}\text { Aserción de la } \\
\text { verificación }\end{array}$ \\
\hline Actuar & $\begin{array}{l}\text { Regulación de } \\
\text { los } \\
\text { componentes } \\
\text { del proyecto }\end{array}$ & $\begin{array}{l}\text { Planeación, } \\
\text { monitoreo y } \\
\text { ejecución de los } \\
\text { componentes del } \\
\text { proyecto }\end{array}$ & $\begin{array}{l}\text { Implementación } \\
\text { de las lecciones } \\
\text { aprendidas en } \\
\text { proyectos } \\
\text { futuros, o en una } \\
\text { reestructuración } \\
\text { del mismo }\end{array}$ \\
\hline
\end{tabular}

Tabla 4 Metodología para la gestión ambiental

\begin{tabular}{|c|c|c|c|}
\hline \multicolumn{4}{|c|}{ Componente: Gestión ambiental } \\
\hline Etapas & $\begin{array}{l}\text { Herramientas y } \\
\text { cnicas }\end{array}$ & Metodologías & $\begin{array}{l}\text { Resultados } \\
\text { esperados }\end{array}$ \\
\hline \multirow{4}{*}{$\begin{array}{l}\text { Planea- } \\
\text { ción }\end{array}$} & $\begin{array}{l}\text { Manual } \\
\text { ambiental de la } \\
\text { entidad }\end{array}$ & $\begin{array}{l}\text { Realización y } \\
\text { utilización del } \\
\text { manual } \\
\text { ambiental } \\
\text { establecido por } \\
\text { la entidad }\end{array}$ & $\begin{array}{l}\text { Aplicación de } \\
\text { buenas prácticas } \\
\text { ambientales en la } \\
\text { sociedad en } \\
\text { general }\end{array}$ \\
\hline & $\begin{array}{l}\text { Estrategias } \\
\text { para que se } \\
\text { haga un } \\
\text { balance } \\
\text { ambiental en la } \\
\text { sociedad }\end{array}$ & $\begin{array}{l}\text { Realizar } \\
\text { capacitaciones } \\
\text { a todos los } \\
\text { interesados } \\
\text { para dar a } \\
\text { conocer la } \\
\text { importancia del } \\
\text { buen manejo } \\
\text { ambiental }\end{array}$ & $\begin{array}{l}\text { Balance óptimo } \\
\text { de buenas } \\
\text { prácticas } \\
\text { ambientales }\end{array}$ \\
\hline & $\begin{array}{l}\text { Implementación } \\
\text { de estrategias } \\
\text { sostenibles }\end{array}$ & $\begin{array}{l}\text { Elaborar un plan } \\
\text { de } \\
\text { sostenibilidad } \\
\text { para la entidad y } \\
\text { el entorno }\end{array}$ & $\begin{array}{l}\text { Generación de } \\
\text { capacidades y } \\
\text { aprobación del } \\
\text { proyecto por } \\
\text { parte de los } \\
\text { interesados } \\
\end{array}$ \\
\hline & $\begin{array}{l}\text { Fomentación } \\
\text { de buenas } \\
\text { prácticas } \\
\text { ambientales }\end{array}$ & $\begin{array}{l}\text { Realizar un plan } \\
\text { ambiental que } \\
\text { pueda ser } \\
\text { replicable por } \\
\text { otras entidades }\end{array}$ & $\begin{array}{l}\text { Aplicable a otros } \\
\text { grupos }\end{array}$ \\
\hline Etapas & $\begin{array}{l}\text { Herramientas y } \\
\text { técnicas }\end{array}$ & Metodologías & $\begin{array}{l}\text { Resultados } \\
\text { esperados }\end{array}$ \\
\hline $\begin{array}{l}\text { Planea- } \\
\text { ción }\end{array}$ & $\begin{array}{l}\text { Proyectos de } \\
\text { innovación } \\
\text { ambiental y } \\
\text { buenos tratos } \\
\text { del ambiente }\end{array}$ & $\begin{array}{l}\text { Realizar } \\
\text { concursos con } \\
\text { proyectos } \\
\text { propuestos por } \\
\text { la comunidad } \\
\text { para la }\end{array}$ & $\begin{array}{l}\text { Nuevas } \\
\text { tendencias y } \\
\text { formas de crear } \\
\text { un medio } \\
\text { ambiente sano y } \\
\text { sostenible }\end{array}$ \\
\hline
\end{tabular}

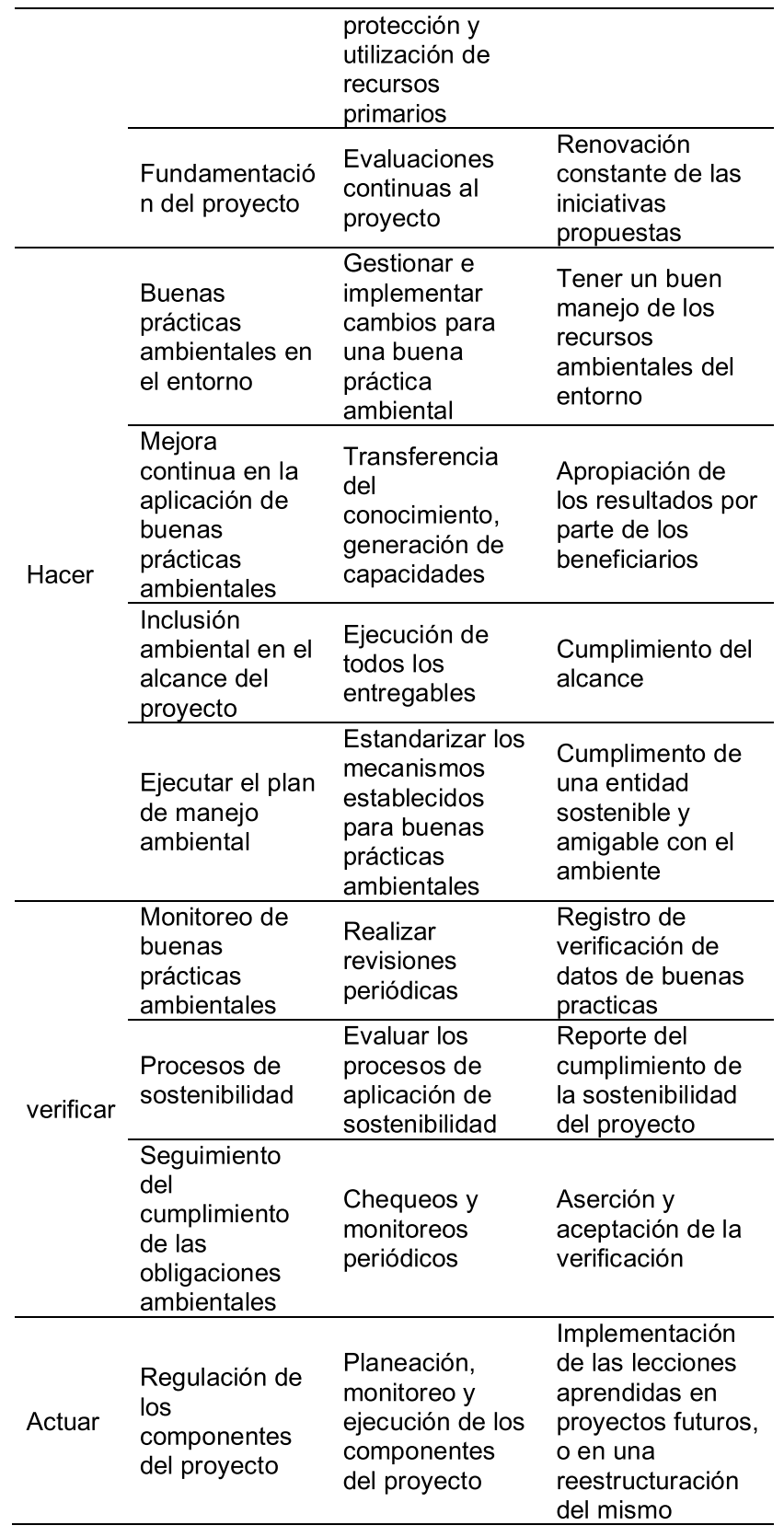

Tabla 5 Metodología para la gestión sociolaboral con los integrantes de la organización

\begin{tabular}{|c|c|c|c|}
\hline \multicolumn{4}{|c|}{$\begin{array}{c}\text { Componente: Gestión sociolaboral con las personas que } \\
\text { integran la organización }\end{array}$} \\
\hline Etapas & $\begin{array}{l}\text { Herramientas y } \\
\text { técnicas }\end{array}$ & Metodologías & $\begin{array}{l}\text { Resultados } \\
\text { esperados }\end{array}$ \\
\hline $\begin{array}{l}\text { Planea- } \\
\text { ción }\end{array}$ & $\begin{array}{l}\text { Balance socio- } \\
\text { laboral }\end{array}$ & $\begin{array}{l}\text { Muestreo } \\
\text { estadístico de } \\
\text { cómo está la } \\
\text { organización }\end{array}$ & $\begin{array}{l}\text { Definición de } \\
\text { la } \\
\text { organización } \\
\text { en su }\end{array}$ \\
\hline
\end{tabular}


Morelos Gómez, J., García Díaz, H. J., \& Prada Sánchez, M. (2020). Dimensiones estratégicas para el fortalecimiento de la responsabilidad social empresarial en Pymes del sector de la construcción en Colombia. Teknos Revista Científica, 20(1), 43-51.

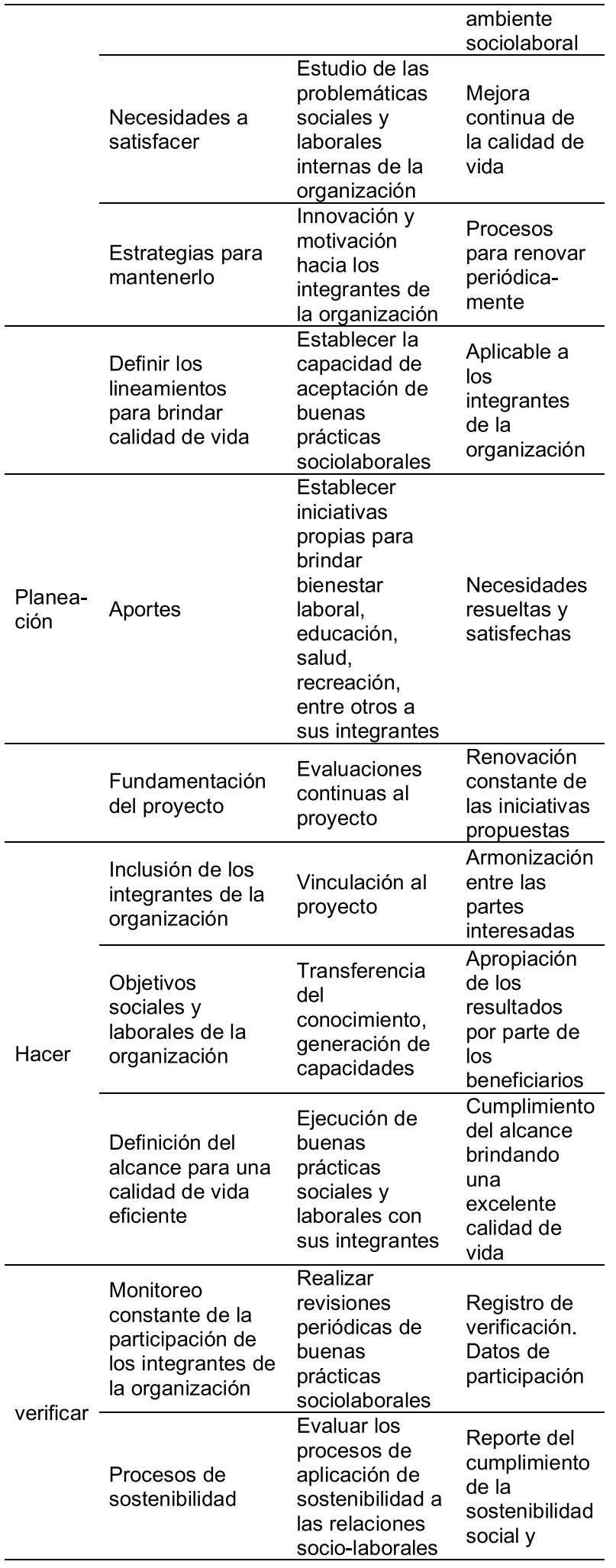

\begin{tabular}{|c|c|c|c|}
\hline & & & \multirow{2}{*}{$\begin{array}{l}\text { laboral del } \\
\text { proyecto } \\
\text { Aserción de } \\
\text { la verificación }\end{array}$} \\
\hline & $\begin{array}{l}\text { Seguimiento del } \\
\text { cumplimiento de } \\
\text { las obligaciones }\end{array}$ & $\begin{array}{l}\text { Chequeos y } \\
\text { monitoreos } \\
\text { periódicos }\end{array}$ & \\
\hline Actuar & $\begin{array}{l}\text { Regulación de los } \\
\text { componentes del } \\
\text { proyecto }\end{array}$ & $\begin{array}{l}\text { Planeación, } \\
\text { monitoreo y } \\
\text { ejecución de } \\
\text { los } \\
\text { componentes } \\
\text { del proyecto }\end{array}$ & $\begin{array}{l}\text { Implementa- } \\
\text { ción de las } \\
\text { lecciones } \\
\text { aprendidas } \\
\text { en proyectos } \\
\text { futuros, o en } \\
\text { una } \\
\text { reestructura- } \\
\text { ción del } \\
\text { mismo }\end{array}$ \\
\hline
\end{tabular}

\section{Conclusiones}

Los resultados de evaluación de implementación de las Pymes en Colombia, mostraron crecimiento en cuanto a la aplicación de las normas de RSE. Se destaca el incremento en los niveles de credibilidad, confianza en el mercado y competitividad del sector de estas pequeñas y medianas empresas que aportaron significativos beneficios a todo el grupo de interés.

Asimismo, se encontró que para los años 2017 y 2018, el porcentaje de las Pymes que aplicaron norma GTC -180, correspondió al $28,8 \%$, las cuales mejoraron sus prácticas dada la motivación y confianza en la implementación de las estrategias de RSE al interior de las mismas.

Se observó también, que el grupo de las Pymes del sector construcción evaluadas, han ampliado su espectro de participación directa con la comunidad, creando conciencia cada vez más, por la gestión del medio ambiente y el desarrollo de programas sociales, lo cual ha hecho que los efectos del impacto social y ambiental, sean cada vez más notorios, dado que se han convertido en pilares para el buen manejo de los recursos.

Finalmente, se tiene que las estrategias $y$ metodologías propuestas por este artículo, aportarán a que las Pymes del sector de la construcción, tengan una guía básica para empezar a generar las bases que le permitan implementar políticas o lineamientos de RSE, partiendo del ciclo propuesto, planear-hacerverificar-actuar- PHVA, y pueda así gestionar la mayor parte de los componentes abarcados por éstas $\mathrm{y}$, del 
mismo modo, direccionarse hacia las buenas prácticas de la gestión social y medioambiental.

Agradecimientos. Universidad de Cartagena, Facultad de Ingeniería, Programa de Especialización en Gerencia de Proyectos de Construcción.

\section{Referencias}

Aguilera, A. \& Puerto, D.P. (2012). Crecimiento empresarial basado en la Responsabilidad Social. Pensamiento \& gestión, $\quad 32(1), \quad 1-26$. http://www.scielo.org.co/scielo.php?pid=S1657$62762012000100002 \&$ script=sci_arttext\&tlng=en

Aparicio, J. \& Valdés, B. (2009). Sobre el concepto de responsabilidad social de las empresas: Un análisis europeo comparado. Cuaderno de Relaciones Laborales. 27(1), 23-75. https://core.ac.uk/download/pdf/38812066.pdf

Benbeniste, S. (2002). El alcance del concepto de la responsabilidad social corporativa de acuerdo a los organismos internacionales promotores del tema. ESADE.

http://www.cyta.com.ar/biblioteca/bddoc/bdlibros/rse/3 34_rsc.pdf

Benítez Sánchez, R. (2012). La responsabilidad social empresarial en las pymes colombianas. Cartagena. https://repositorio.unicartagena.edu.co/handle/11227/ 1217

Botero Botero, L. F. (2009). Responsabilidad social empresarial en el sector de la construcción. Medellín. http://publicaciones.eafit.edu.co/index.php/administer/ article/view/264

Camacho, J. I. (2015). Las normas de responsabilidad social. Su dimensión en el ámbito laboral de las empresas. Revista latinoamericana de derecho social, $1(20)$,

3-29.

http://www.scielo.org.mx/scielo.php?script=sci_arttext \&pid=S1870-46702015000100003

CAMACOL. (2019). Premio a la responsabilidad social. Colombia. https://camacol.co/comunicados/camacolhizo-entrega-de-los-premios-la-responsabilidad-social

Centro Colombiano de Responsabilidad Social. (2004). CCRS. Colombia.

Comisión de las comunidades Europeas (2001). Green Book, Fomentar un marco europero para la responsabilidad social de las empresas (CCE). Bruselas.

Correa Jaramillo, J. G. (2007). Evolución histórica de los conceptos de responsabilidad social empresarial y balance social. 10(20), 87-102. http://www.scielo.org.co/pdf/seec/v10n20/v10n20a6.p df

Cumbre de Lisboa. (Marzo de 2000). Cumbre Lisboa. Obtenido de www.europa.eu.int/comm/employment_social/socdial/csr/csr_commissionsact.html

Duque, Y.V. \& Martínez, D. (2011). Responsabilidad social empresarial en la dimensión laboral: caso Bancolombia y BBVA. Revista Facultad de Ciencias Económicas, 20(1), 171-187. http://www.scielo.org.co/scielo.php?pid=S0121$68052012000100011 \&$ script=sci_abstract\&tIng=es

Escuela de Negocios (2005). Integración de la responsabilidad social de la empresa en la gestión de las pymes y su incidencia en el empleo. España. http://media.eoi.es/nw/Multimedia/EstudiosFSE/2005 _02_estudiocompleto.pdf

Fundación Entorno, c. e. (2009). La responsabilidad social corporativa y las constructoras medianas. España. https://upcommons.upc.edu/handle/2099.1/5661

García, R. F. (2013). La dimensión económica del desarrollo sostenible. Editorial Club Universitario. https://www.upb.edu.co/es/proyeccionsocial/sostenibilidad/dimension-economica-ods

Grueso, Merlin. 2009. Responsabilidad social empresarial e igualdad en cuestiones de empleo. Universidad y Empresa, $\quad 17(1), \quad 244 \quad$ - 251. https://revistas.urosario.edu.co/index.php/empresa/art icle/view/1091/989

ICONTEC. (2008). GTC 180. En ICONTEC, Guía Técnica Colombiana (pág. E: Guidance on social responsability). Bogotá, D.C.: Instituto Colombiano de Normas Técnicas y Certificación ICONTEC.

Mababu, R. (2010). Actitudes de los empresarios directivos hacia la responsabilidad social. Revista de psicología del trabajo y de las organizaciones. 26(2), 101-114. http://scielo.isciii.es/scielo.php?script=sci_arttext\&pid =S1576-59622010000200002

Martí-Noguera, J. J., Puerta-Lopera, I. C. y Rojas-Román, P. (2017). A propósito de la Responsabilidad Social Universitaria. Revista Colombiana de Ciencias Sociales, 8(2), 294-301. http://dx.doi.org/10.21501/22161201.2291

Morelos, J. y Fontalvo, T. J. (2014). Análisis de los factores determinantes de la cultura organizacional en el ambiente empresarial. Entramado, 10(1), 96-105. https://www.redalyc.org/pdf/2654/265431574006.pdf

Olano Isaza, A. (2014). Estudio de responsabilidad social en 
Morelos Gómez, J., García Díaz, H. J., \& Prada Sánchez, M. (2020). Dimensiones estratégicas para el fortalecimiento de la responsabilidad social empresarial en Pymes del sector de la construcción en Colombia. Teknos Revista Científica, 20(1), 43-51.

Colombia, un compromiso de todos. Bogotá. https://repository.unimilitar.edu.co/bitstream/handle/1 0654/12615

Osorio Cuero, L. M. (2012). Análisis de las RSE en la constructora Mecón S.A. en la ciudad de Cali. Bogotá. https://repository.javeriana.edu.co/handle/10554/1109 6 